\title{
Resources for selecting popular music recordings
}

\author{
Eamon Tervell \\ Moore College of Art and Design, Philadelphia, Pennsylvania, USA
}

\begin{abstract}
Purpose - The purpose of this paper is to assist music selectors at academic and public libraries by providing resources useful for selecting popular sound recordings, while emphasizing the ongoing need for monitoring trends and new releases in popular music.

Design/methodology/approach - This paper identifies key resources to aid the selection of popular music recordings, beginning with the challenges unique to popular music. The resources are divided into four major sections: online tools, print tools, periodical tools, and staying current.

Findings - Popular music is widely collected by libraries, yet selection and acquisition is made difficult by the numerous genres and constant rotation of popular artists and albums. The utilization of a variety of online and print resources is ideal in order to build or maintain a well-stocked popular music collection.

Originality/value - This paper offers guidance to librarians responsible for sound recordings. No previous discussions of music selection have considered popular music as a whole, while popular music recordings are widely collected by libraries and require a great deal of currency.
\end{abstract}

Keywords Collections management, Selection, Music, Popular culture

Paper type General review

Popular music is widely collected by libraries, yet maintaining a quality collection can be a challenging prospect for professionals lacking awareness of popular music or selecting recordings. Popular music recordings encompass an entire world of genres and performers and are available on a number of formats. Unfamiliarity with the proper resources is the most significant hurdle for librarians new to selecting and acquiring popular music. Selectors must remain aware of the constantly revolving cast of popular artists, new releases, and trends within genres, a task requiring significant effort. Considering these issues, it is remarkably easy to feel overwhelmed in selecting recordings for one's collection. This paper provides an overview of online and print aids available to make the selection of popular music a less daunting proposition.

Due to the number of factors at hand, it is important to first review the collection development policy, evaluate the existing collection, and consider the needs of the patrons served by the library. The collection development policy will to a large degree determine which materials are acquired and which do not meet the selection criteria. Time used to review the policy is time well spent, as it is an essential first step in successfully selecting recordings. During the collection evaluation stage it is recommended to check circulation statistics to gauge patron interest in the existing collection while also noting any gaps in genre or period as the recordings are reviewed. Ideally, the selector will already be familiar with the collection and well informed of its strengths and weaknesses. This familiarity

The current issue and full text archive of this journal is available at www.emeraldinsight.com/0160-4953.htm

Collection Building

29/1 (2010) 27-30

(C) Emerald Group Publishing Limited [ISSN 0160-4953]

[DOI 10.1108/01604951011015277] coupled with evaluation of existing recordings allows the selector to compensate for areas not collected as widely as they should be.

Regarding patron needs, the selector will be able to determine the needs of the community best. For example, would a strong Latin Pop collection be of interest in one's public library, or a range of improvisational jazz be in support of a popular department at one's academic institution? Beyond audience, additional factors to consider are format and quality of the recording, both of which are frequently addressed by album reviews. Only after the collection development policy, the existing recording collection's strengths and weaknesses and patron needs are examined as advised to begin selecting materials using the tools described.

Popular music serves as an umbrella term for any music with a large base of listeners. The term encompasses a wide array of genres, the largest of which include blues, country, easy listening, electronic, folk, jazz, latin, metal and alternative rock, pop, rap and hip-hop, reggae, rhythm and blues, rock, and world. Its worldwide popularity aside, classical music falls outside of the popular music rubric and is in a category of its own, making it outside the scope of this paper. There is a great deal of overlap between popular music genres and they can be almost endlessly subdivided. It is the author's intent that the resources in this paper remain impartial to genre so that they may be as equally useful to a librarian selecting bebop as to one requiring post-punk.

Over the last several years the popularity of online music purchases and downloads has soared, but compact discs are the most common means of listening to music and have remained a highly popular medium for recordings since their introduction in 1982. Digital music is on the rise and many predict online music sales will eclipse that of physical music

Received: September 2009

Accepted: October 2009 
recordings within a decade. On the other hand, compact discs are currently and will continue to be widely used for the foreseeable future. As such, CDs will remain the format of focus throughout this paper.

The resources below are intended to guide those responsible for selecting music at their institution, whether beginning a collection of the essentials or updating existing recordings. The tools will be particularly useful for librarians who do not have prior knowledge of popular music or experience selecting recordings for library collections. While non-specialists are likely to benefit the most, those with a musical background will find practical tools and reminders as well.

\section{Online tools}

There exists a wealth of online resources to aid in selecting recordings. The tools below include directories created by and for collection development and music librarians, as well as selected web sites to aid in locating reviews or listening to clips of popular music. Many sources include music outside of the scope of popular music, and all are available without cost.

\section{Directories}

Music Selection resources on the $W W W$

(www.halcyon.com/aseaberg)

(www.library.yale.edu/cataloging/music/ctwebrt.htm)

Another directory useful for the selector of sound recordings is "Music and Film Resources on the Web for Librarians." Created by Mickey Koth at the Yale University Music Library, this resource provides excellent starting points for reviews and "Best Of" lists, and includes web sites recommended for purchasing CDs. While the "General music resources" and "Collection development \& acquisitions" sections are of primary interest, the web site also includes links for librarians and catalogers working with film and video. Genre coverage includes all major music and is especially strong in jazz.

\section{Review sources}

Reviews are critical tools, providing selectors with context and a recommendation on the item at hand. The most comprehensive sources for reviews of general sound recordings - that is, not limited to specific genres or regions - are "All Music", "Billboard", and "Barnes \& Noble Music". Review sources can be broken down into two major categories: "Reference" and "Retailers". Each source has its strengths and weaknesses in types of music covered, but "All Music" is widely accepted to be the best online database containing reviews, and Billboard as the best source for charts of top-selling albums and songs.

\section{Reference \\ All Music \\ (www.allmusic.com)}

All Music is impressive in its scope and quality, and any given entry for an artist or recording features a helpful set of links to discography, biography, similar artists, and more. The pages for many albums are likewise full of information, including release date, track listings, and a review. A feature ideal for music selectors is the genre navigation bar, allowing users to select major genres, browse hundreds of sub-genres, and view defining albums. Brief excerpts of tracks from many artists can be listened to by searching for an artist, album or song. Of particular interest to those seeking background on significant genres are the "Related Essays" under each major genre section. While the Electronica and R\&B sections are slightly lacking in coverage, this source remains highly recommended for all popular music genres.

\section{Billboard}

(www.billboard.com)

Billboard is highly recommended to librarians collecting popular music. This source features the Billboard 200, a list of the week's top-selling albums and a good indicator of a given artist's popularity. Billboard includes brief reviews of new releases in every major genre, assigning a score to each album for quick reference of its critical reception. Major sections of the web site include "Charts," "News," and "New releases." Users can search for artists, albums, songs, or news in Billboard's comprehensive database, but to listen to a track the MP3 must first be purchased. Site navigation can be difficult when looking for genres. Selectors seeking to remain in the loop regarding music industry news can elect to receive Billboard's daily e-mail. For information on current popular recordings one can do no better.

\section{HotList}

(http://cdhotlist.btol.com)

Written by librarians for librarians, this Baker \& Taylor sponsored resource features monthly reviews of new releases. Popular music genres addressed by reviewers include Country/Folk, Jazz, Rock/Pop, and World/Ethnic. While the genre distinctions are quite broad and reviews rarely run longer than a paragraph, CD HotList provides bibliographic information and context for highlighted new releases and is useful as a librarian-oriented source for reviews. The web site does not offer a search function but reviews from 2004 forward are archived. Sound clips from the albums reviewed are not available.

\section{Retailers}

Barnes \& Noble Music

(http://music.barnesandnoble.com)

This retailer is better known for its book and film selection than for music, yet their online store contains features especially helpful for the sound recording selector. Many titles have lengthy sound clips combined with reviews from All Music Guide, so one may read about a particular item while hearing it. These features are not lost on those whom often resort to selecting auditory material based solely on reviews, rarely able to hear a recording before purchasing it. Barnes \& Noble Music also offers recommended lists such as "Critics Choice" and "Ultimate CD Collection," which are of use if the selector requires basic titles. Finally, the Advanced Search allows for fine-grained options, including Keywords, Format, and Style.

\section{Amazon}

(www.amazon.com)

Amazon is quite similar to Barnes \& Noble Music in terms of search and browsing options, and the web site can be difficult to navigate with the clutter of user reviews, discounts, and "Best Of" lists that may or may not correspond to what the selector seeks. However, Amazon's recommendation system for related titles is highly accurate for popular items. As such, browsing similar items serves as an excellent finding aid for 
related recordings. Despite navigational drawbacks Amazon remains an important source to consider when making decisions for acquisitions, and it is particularly useful for locating and purchasing used recordings that are out of print.

\section{Print tools}

Not all resources to aid in selecting sound recordings are available electronically. While the Reference and Retailers web sites listed above offer unique resources, there is rarely anything as useful as a well-organized and comprehensive printed work on the subject. Although only two monographs exist to assist the selection or acquisition of sound recordings, both are high quality resources for the sound recordings selector.

\section{Library Acquisition of Music}

An outstanding title detailing the "how-to" of selecting music recordings is Library Acquisition of Music (Scarecrow Press, 2004). Part of the Music Library Association's Basic Manual Series, this volume focuses on how to acquire music materials, including such chapters as techniques for ordering music, managing approval plans, locating out of print items, and a directory of vendors with regard to musical specialty. The book attends to the publishing of both printed and recorded music in separate chapters.

\section{A Basic Music Library: Essential Scores and Recordings}

The selector seeking a guide on general acquisitions procedures should locate A Basic Music Library: Essential Scores and Recordings, also from the Music Library Association. This title is the most comprehensive print source for choice recordings that are the staple of many music collections. Chapters 20 through 33 will be of particular interest to selectors desiring classic popular music from various time periods and locales. While needing an update, classic recordings fortunately do not call for frequent revision. Music selectors interested in developing historically popular music collections will find A Basic Music Library to be of tremendous value, yet those seeking today's popular albums will want to instead consult the sources listed in the Reference and Retailers sections above. This volume is also highly useful for comparing titles to locate strong and weak points of the collection, thus giving the selector an idea of which area to collect in.

\section{Periodical tools}

A selector need not remain within specialized resources for choosing music recordings, whether online or in print. Resources used more often for print materials and common by collection development standards may also be used for selecting music. These tools include Booklist and Library Fournal. Booklist, for example, publishes the valuable Editor's Choice list annually, which includes an audio section. Library Fournal offers the column, "Music for the Masses," which describes defining recordings in popular music sub-genres. While the number of recordings that are reviewed is less substantial than in other tools, these library-specific sources ought not be overlooked.
Many popular music periodicals include album reviews in both print and online editions. Below are magazines selected for their reliable reviews, intended as an introduction to each popular music genre. Blues and R\&B: Blues and Rhythm (www.bluesandrhythm.co.uk); Fazz \& Blues Report (www.jazz-blues.com)

- Country: Country Weekly (www.countryweekly.com/ reviews)

- Electronic: The Wire (www.thewire.co.uk)

- Folk: Dirty Linen (www.dirtylinen.com); Sing Out! (www.singout.org/magazine.html)

- Jazz: Downbeat Magazine (www.downbeat.com); JazzTimes (http://jazztimes.com/sections/albums/articles)

- Latin: Descarga (www.descarga.com); Latin Beat (www.latinbeatmagazine.com/reviews.html)

- Metal and Alternative Rock: Alternative Press (www.altpress.com/reviews); Decibel (http:// decibelmagazine.com)

- Pop and Rock: Rolling Stone (www.rollingstone.com/ reviews); Spin (http://spin.com/reviews)

- Rap and Hip Hop: HipHopDX (www.hiphopdx.com/ index/reviews); XXL Magazine (www.xxlmag.com)

- Reggae: The Beat (www.getthebeat.com); United Reggae (www.unitedreggae.com)

- Rock: New Music Exchange (www.nme.com/reviews/ albums); Pitchfork (http://pitchfork.com/reviews/recent)

- World: Roots World (www.rootsworld.com/rw); Songlines (www.songlines.co.uk)

\section{Staying current}

Keeping up with top artists, albums, and genres is a crucial component of selecting popular recordings. Currency ensures that the collection is able to reflect the rapid rotation of charttopping musicians and albums. In addition to the sources for reviews listed above, these resources will assist selectors in remaining up to date with new releases and developments in the world of popular music.

\section{Notes}

(www.musiclibraryassoc.org/publications/notes.shtml)

The Music Library Association's quarterly journal Notes offers much in the way of remaining in the music publishing loop. Containing reviews of music-related materials, information from vendors on recent releases, and well-researched articles, it is a wealth of information for the music selector. Particularly useful for acquisitions and selection are "Music received," "Music publisher's catalogs," and "Index to advertisers." These sections compile new music publications, announcements of new catalogs and contact information for music publishers, and the advertisers that appear in the publication, respectively. All are excellent tools for remaining informed and up to date in music publication.

\section{Last.fm}

(www.last.fm)

Last.fm is a unique aid for remaining current with popular music. Last.fm provides music listeners with a social web experience, allowing users to build a profile, listen to and recommend songs, and connect with others based on musical interests. Particularly valuable for librarians is the option to search for artists and find not only recommendations for 
similar musicians, but to peruse an alternative to Billboard's charts. Last.fm's weekly artist charts track top musicians based on the number of Last.fm listeners, instead of salesbased rankings such as Billboard. Users have an array of searching and browsing options including genre and decade. While Last.fm includes all genres on its web site, coverage leans heavily towards rock in accordance with its users' preferences.

\section{Music Library Association E-mail Listserv \\ (www.musiclibraryassoc.org/resources/mla-l.shtml)}

The Music Library Association's e-mail listserv MLA-L is an excellent way to connect with colleagues and keep abreast of developments in selecting music materials. There are approximately 1,100 members of the group. While the postings address all aspects of music librarianship, collection development and acquisitions are frequently discussed. Instructions for subscribing are located at the web site above. Archived postings, another handy resource if one has a specific question to be answered that was previously discussed, can be found at http://listserv.indiana.edu/ archives/mla-1.html This listserv is active but does not overburden one's inbox, and often contains discussions relevant to collection development and acquisitions. MLA-L also acts as a conduit for music library news, so one can stay informed of new developments without major time expenditure.

\section{Conclusion}

While a one-stop resource for selecting music recordings would indeed be useful, it does not exist. When considering recordings to add to one's collection, consider the collection development policy, strengths and weaknesses of the existing collection, and patron needs before immersing oneself in the world of sound recordings. There are many online, print and periodical sources to aid in selecting recordings, and combining these tools with one another is the best method for assembling a high quality popular music collection. Finally, remain current using resources from the Music Library Association and user-driven music web sites. Selecting popular sound recordings may be intimidating, but with the assistance of these resources the process will become second nature.

\section{About the author}

Eamon Tewell is Senior Library Assistant at Moore College of Art and Design, where he is responsible for acquisitions and collection maintenance. He received his MLIS from Drexel University. Eamon Tewell can be contacted at: etewell@moore.edu. 